MATHEMATICS OF COMPUTATION

Volume 70, Number 236, Pages 1737-1744

S $0025-5718(01) 01327-8$

Article electronically published on May 11, 2001

\title{
FINDING PRIME PAIRS WITH PARTICULAR GAPS
}

\author{
PAMELA A. CUTTER
}

\begin{abstract}
By a prime gap of size $g$, we mean that there are primes $p$ and $p+g$ such that the $g-1$ numbers between $p$ and $p+g$ are all composite. It is widely believed that infinitely many prime gaps of size $g$ exist for all even integers $g$. However, it had not previously been known whether a prime gap of size 1000 existed. The objective of this article was to be the first to find a prime gap of size 1000, by using a systematic method that would also apply to finding prime gaps of any size. By this method, we find prime gaps for all even integers from 746 to 1000 , and some beyond. What we find are not necessarily the first occurrences of these gaps, but, being examples, they give an upper bound on the first such occurrences. The prime gaps of size 1000 listed in this article were first announced on the Number Theory Listing to the World Wide Web on Tuesday, April 8, 1997. Since then, others, including Sol Weintraub and A.O.L. Atkin, have found prime gaps of size 1000 with smaller integers, using more ad hoc methods. At the end of the article, related computations to find prime triples of the form $6 m+1,12 m-1,12 m+1$ and their application to divisibility of binomial coefficients by a square will also be discussed.
\end{abstract}

\section{INTRODUCTION}

In $1928, D$. H. Lehmer realized that if $p-1=F R$ where $F>p^{1 / 2}$ is factored, then there is a quick, practical way to show if $p$ is prime. In 1975, this result was extended by Lehmer, Brillhart and Selfridge [1, so that one only needs to have the factored part $F>p^{1 / 3}$ to get a quick test. The test is based on the following theorem.

Theorem 1. Let $N-1=F R$, where $F$ is factored, $F>N^{1 / 3}$ and $(F+1)^{2} \neq N$. Assume that, for each prime $p_{k}$ dividing $F$, there exists an $a_{k}$ such that

(i) $a_{k}^{N-1} \equiv 1(\bmod N)$, and

(ii) $\operatorname{gcd}\left(a_{k}^{\frac{N-1}{p_{k}}}-1, N\right)=1$.

Write $R=2 F s+r$ where $1 \leq r<2 F$. Then $N$ is prime if and only if $s=0$ or $r^{2}-8 s$ is not the square of an integer.

To apply this to the prime gap problem, we need to have partial factorizations of $p-1$ and $p+g-1$. To do this, we proceed as follows: For a given even integer $g$, we select $a$ and $b$ to be the largest integers with $2^{a}, 3^{b}<e^{g / 4}$. We select $p_{0}$ to be the

Received by the editor February 23, 1999.

2000 Mathematics Subject Classification. Primary 11A41; Secondary 11Y11, 11Y55, 11Y99.

Key words and phrases. Prime numbers, prime gaps, consecutive primes, prime triples. 
least positive integer $\left(\bmod 2^{a} 3^{b}\right)$ as follows, by the Chinese Remainder Theorem:

if $g \equiv 2(\bmod 3)$, then $p_{0} \equiv 1 \quad\left(\bmod 2^{a}\right)$ and $p_{0}+g \equiv 1\left(\bmod 3^{b}\right)$,

if $\quad g \not \equiv 2(\bmod 3)$, then $p_{0} \equiv 1 \quad\left(\bmod 3^{b}\right)$ and $p_{0}+g \equiv 1 \quad\left(\bmod 2^{a}\right)$.

We need two cases, for, if $g \equiv 2(\bmod 3)$ and we select $p_{0}\left(\bmod 2^{a} 3^{b}\right)$ such that $p_{0} \equiv 1\left(\bmod 3^{b}\right)$ and $p_{0}+g \equiv 1\left(\bmod 2^{a}\right)$, then we would have that 3 divides $p_{0}+g$. If we were to only select $p_{0}\left(\bmod 2^{a} 3^{b}\right)$ such that $p_{0} \equiv 1\left(\bmod 2^{a}\right)$ and $p_{0}+g \equiv 1\left(\bmod 3^{b}\right)$, then we would have that 3 divides $p_{0}$ when $g \equiv 1(\bmod 3)$. Selecting $p \equiv p_{0}\left(\bmod 2^{a} 3^{b}\right)$ with $p<\min \left(2^{3 a-1}, 3^{3 b-1}\right)$, we get $p-1$ and $p+g-1$ divisible by $2^{a}$ and $3^{b}$ (the order depending on $\left.g(\bmod 3)\right)$. Notice that $2^{a}$ and $3^{b}$ are factors $>(p+g)^{1 / 3}>p^{1 / 3}$. Thus we can use Theorem 1 above to determine whether $p$ and $p+g$ are prime.

In addition to the prime gap problem, Sections 4 and 5 will show how Theorem 1] can be applied to find prime triples of the form $6 m+1,12 m-1,12 m+1$. Prime triples of this form will be used to complete the proof of the following theorem:

Theorem 2. $\left(\begin{array}{c}2 n \\ n\end{array}\right)$ is divisible by the square of a prime $p \geq \sqrt{n / 5}$ for all $n \geq 2082$.

\section{The ALGORITHM}

The algorithm used to find consecutive primes with a given gap $g$ is as follows. Step 1: Find $p_{0}, 2^{a}$ and $3^{b}$ as stated above.

Step 2: The numbers $p$ that we will be testing are of the form $p=p_{0}+i 2^{a} 3^{b}$, as $i$ runs from 1 to $10^{6}$. We do not actually want to put all of these numbers through the primality test, as some of them may be divisible by small primes. We will sieve these numbers out as follows: For each prime $q$ up to $10^{4}$, if $i$ is in either of the residue classes

$$
\frac{-p_{0}}{2^{a} 3^{b}} \quad(\bmod q) \text { or } \frac{-p_{0}-g}{2^{a} 3^{b}} \quad(\bmod q)
$$

then either $p$ or $p+g$, respectively, will be divisible by $q$. So we will discard this value of $i$. The remaining $i$ 's we store in a table, which we call $T_{1}(g)$.

Step 3: For each value of $i$ in $T_{1}(g)$, we use Theorem 1 to test if $p=p_{0}+i 2^{a} 3^{b}$ is a prime. To do this, we start with $a_{k}=2$. If, along with the other hypotheses, conditions (i) and (ii) of Theorem 1 are met, we have a prime. If they are not satisfied, we let $a_{k}$ run through primes, up to the limit 255 . If we reach this limit with no success in finding an $a_{k}$, or proving $p$ composite, then we give up trying to determine primality of this value $p$ and move on to the next $i$ in $T_{1}(g)$. There are three ways we will learn that $p$ is composite from our algorithm:

- $a_{k}{ }^{p-1} \not \equiv 1 \quad(\bmod p)$

- $1<\operatorname{gcd}\left(a_{k}{ }^{(p-1) / b}-1, p\right)<p$ where $b$ is 2 or 3 , depending on $p$

- In the theorem, we have $s>0$ and $r^{2}-8 s$ is the square of an integer

If we find an $a_{k}$ which satisfies conditions (i) and (ii) of Theorem 1 we then perform the same test with $p+g$. If we have primality of both $p$ and $p+g$, we store the values $p, p+g$ and the respective $a_{k}$ 's in a table, call it $T_{2}(g)$.

Step 4: For every prime pair $p, p+g$ in $T_{2}(g)$, we need to determine whether the numbers $p+j$, as $j$ runs from 1 to $g-1$, are composite. We do this in two steps. 
(a) We first check if the numbers $p+j$ are divisible by primes up to $10^{4}$. For each prime $q<10^{4}$, whenever $j \equiv-p(\bmod q)$, we have $p+j$ divisible by $q$. Thus $p+j$ is composite. We store the remaining $j$ 's (those where $p+j$ is not divisible by a prime $<10^{4}$ ) in a table $T_{3, p}(g)$.

(b) For each $j$ in $T_{3, p}(g)$, we run at least one pseudoprime test on $n=p+j$. If $n$ is not a pseudoprime to either base 2 or 3 , then we know it is composite and we go on to the next $j$ in $T_{3, p}(g)$. If $n$ should happen to pass pseudoprime tests to both the bases 2 and 3, we say $n$ is probably prime and we disregard this prime pair $p$, $p+g$. We then move to the next prime pair $p, p+g$ in $T_{2}(g)$. If all of the numbers $p+j$ where $j=1, . ., g-1$, are not pseudoprimes to either base 2 or 3 , then they are all composite, and we have found a consecutive pair of primes with gap $g$, as desired.

\section{EXPECTED VALUES}

Let $\pi_{g}(x)$ be the number of primes $n$ up to $x$ such that $n+g$ is also prime. Then we have the following conjecture, due to Hardy and Littlewood [3].

Conjecture 1. If $g$ is even, then

$$
\pi_{g}(x) \sim \alpha \prod_{\substack{p \mid g \\ p>2}} \frac{p-1}{p-2} \cdot \frac{x}{\log ^{2} x}
$$

where

$$
\alpha=2 \prod_{p>2} \frac{\left(1-\frac{2}{p}\right)}{\left(1-\frac{1}{p}\right)^{2}} .
$$

If we first just assume that the primality of the two numbers $n, n+g$ near $x$, randomly chosen, are independent events, then we would have

$$
\pi_{g}(x) \sim \frac{x}{\log ^{2} x} .
$$

But we know these events are not independent, so we correct this argument by multiplying by the factor

$$
\prod_{p} \frac{\left(1-\frac{\omega(p)}{p}\right)}{\left(1-\frac{1}{p}\right)^{2}}
$$

since the probablity that two random integers are not divisible by $p$ is $\left(1-\frac{1}{p}\right)^{2}$, and the probablity that our particular numbers are not divisible by $p$ is $\left(1-\frac{\omega(p)}{p}\right)$, where $\omega(p)$ is the number of solutions to $n(n+g) \equiv 0(\bmod p)$. Noting that $\omega(p)=2$ unless $p$ divides $g$, whence $\omega(p)=1$, we obtain the stated conjecture (with some rearrangement).

Recall that, in Step 2 of our algorithm, we did not want to put all of our numbers $p$ through the primality test. So we sieved out the values of $p$ which were divisible by primes up to $10^{4}$. We can thus make a further adjustment to our expected values to account for this, and we arrive at the following: 
Heuristic 1. Knowing that the numbers $n, n+g$ are not divisible by primes less than $10^{4}$, we have, assuming $g<10^{4}$, that the probability that $n$ and $n+g$ near $x$ are both prime is $\approx \beta \frac{1}{\log ^{2} x}$ where $\beta=\prod_{p<10^{4}} \frac{1}{\left(1-\frac{1}{p}\right)^{2}} \approx 269.76$.

We justify the heuristic as follows:

We previously had that the probability that $n$ and $n+g$ are both prime was

$$
\begin{aligned}
& \sim \prod_{\substack{p \\
\omega(p) \neq 2}} \frac{\left(1-\frac{1}{p}\right)}{\left(1-\frac{1}{p}\right)^{2}} \cdot \prod_{\substack{p \\
\omega(p)=2}} \frac{\left(1-\frac{2}{p}\right)}{\left(1-\frac{1}{p}\right)^{2}} \cdot \frac{1}{\log ^{2} x} \\
& =\prod_{p \mid g} \frac{\left(1-\frac{1}{p}\right)}{\left(1-\frac{1}{p}\right)^{2}} \cdot \prod_{p \nmid g} \frac{\left(1-\frac{2}{p}\right)}{\left(1-\frac{1}{p}\right)^{2}} \cdot \frac{1}{\log ^{2} x} .
\end{aligned}
$$

Since we know $n$ and $n+g$ are not divisible by primes up to $10^{4}$, we can make the following adjustment: The probability that $n$ and $n+g$ are both prime is now

$$
\sim \prod_{\substack{p \mid g \\ p>10^{4}}} \frac{\left(1-\frac{1}{p}\right)}{\left(1-\frac{1}{p}\right)^{2}} \cdot \prod_{\substack{p \nmid g \\ p>10^{4}}} \frac{\left(1-\frac{2}{p}\right)}{\left(1-\frac{1}{p}\right)^{2}} \cdot \prod_{p<10^{4}} \frac{1}{\left(1-\frac{1}{p}\right)^{2}} \cdot \frac{1}{\log ^{2} x}
$$

Computing this last product, we get our heuristic, provided the contribution of the first two products here is negligible. Now, if $p>10^{4}$, then $p>g$, so $p \nmid g$. Thus the first product equals 1 ; and it remains to show that $\prod_{p>10^{4}}\left(1-\frac{2}{p}\right) /\left(1-\frac{1}{p}\right)^{2}$ is close to 1 . Now

$$
\begin{aligned}
0 & <-\sum_{\substack{p \text { prime } \\
p>10^{4}}} \log \left(\frac{1-\frac{2}{p}}{\left(1-\frac{1}{p}\right)^{2}}\right)=-\sum_{\substack{p \text { prime } \\
p>10^{4}}} \log \left(1-\frac{1}{(p-1)^{2}}\right) \\
& \leq c \sum_{\substack{p \text { prime } \\
p>10^{4}}} \frac{1}{(p-1)^{2}} \leq c \sum_{n>10^{4}} \frac{1}{(n-1)^{2}} \\
& \leq c \int_{10^{4}}^{\infty} \frac{1}{(x-1)^{2}} d x=c\left[\frac{-1}{x-1}\right]_{10^{4}}^{\infty}=\frac{c}{10^{4}-1}
\end{aligned}
$$

where, since $p \geq 10^{4}+1$, we get $c<1+\frac{1}{10^{8}-1} \approx 1.00000001$.

Thus, taking exponentials of the above,

$$
1 \geq \prod_{p>10^{4}} \frac{\left(1-\frac{2}{p}\right)}{\left(1-\frac{1}{p}\right)^{2}} \geq e^{-c /\left(10^{4}-1\right)} \approx 1-.0001,
$$

so is close to 1 , justifying the heuristic.

Next, we would like to determine how many of these prime pairs $n, n+g$ we would expect to be consecutive. If we have a random number $m$ near $x$, the probability that $m$ is composite is $1-\frac{1}{\log x}$. Assuming independence of compositeness of the $g-1$ numbers between $n$ and $n+g$, the probablity that these numbers are all composite is $\left(1-\frac{1}{\log x}\right)^{g-1}$. Now, for our gaps $g$, the values of $x$ are around $e^{g / 2}$. 
So we would have

$$
\left(1-\frac{1}{\log x}\right)^{g-1} \sim\left(1-\frac{2}{g}\right)^{g-1} \approx e^{-2} .
$$

We thus expect that roughly 1 out of every $e^{2} \approx 7.39$ prime pairs $p, p+g$ in $T_{2}(g)$ should be consecutive primes (i.e., have no primes between $p$ and $p+g$ ). Upon looking at some of the data below, this crude estimate seems to be reasonable.

\section{DAtA}

For each gap $g$, Table 1 (on the next page) contains the exponents $a$ and $b$ of 2 and 3, respectively, and the first value of $i$, which give consecutive primes $p, p+g$, where $p=p_{0}+i 2^{a} 3^{b}$ (recall we have $i$ running from 1 to $10^{6}$ ), and $p_{0}$ is constructed as described by equation (1.1). Also listed is the number of values of $i$ for which consecutive primes $p, p+g$ were obtained. There may be more values, since the algorithm ignores a pair $p, p+g$ if some number $p+j, 1 \leq j \leq g-1$, passes 2 pseudoprime tests; however, it is highly unlikely that there is such a problem with the data. Table 1 is arranged with the original gap of size 1000 listed first, followed by several gaps of size larger than 1000 that were found. It then proceeds numerically, starting with gap 746. Gaps $g$ listed with an asterisk are gaps that were already known to exist at the time these computations were performed ([4], [7], [8]). These gaps were found again just to make the list complete.

\section{Other calculations}

In addition to the prime gap problem, the primality testing ideas of Brillhart, Lehmer and Selfridge can be applied to find prime triples of the form $6 m+1$, $12 m-1,12 m+1$. Prime triples of this form will be used to complete the proof of the following theorem:

Theorem 2. $\left(\begin{array}{c}2 n \\ n\end{array}\right)$ is divisible by the square of a prime $p \geq \sqrt{n / 5}$ for all $n \geq 2082$.

Granville and Ramaré have verified this theorem for $2082 \leq n \leq 10^{10}$ by using a direct consequence of Kummer's theorem, and for $n \geq 2^{1617}$ by using bounds on exponential sums [2]. By using the following proposition of Granville and Ramaré, it becomes a practical computational problem to establish this theorem for $10^{10} \leq$ $n \leq 2^{1617}$.

Proposition 1. If $m$ is a positive integer for which $p=6 m+1, q=12 m-1$, and $r=12 m+1$ are all prime, then at least one of $p^{2}, q^{2}, r^{2}$ divides $\left(\begin{array}{c}2 n \\ n\end{array}\right)$, for each integer $n$ in the interval $\left[96 m^{2}-2 m, 108 m^{2}+3 m-2\right]$, with the one exception, namely $m=1, n=104$.

The biggest difficulty in applying this proposition is in primality proving. In general, when the integers involved are large, it is difficult to prove that $p, q$ and $r$ are all prime in a reasonable amount of time. However, since we have Theorem 1 we can construct the integers $p, q$ and $r$ in a specific manner, to make primality testing easier.

To apply Theorem 1 in finding prime triples $p, q, r$ as in the proposition, we need to have the factorizations of part of $m$ and $6 m-1$. To do this, we proceed as follows: For a given odd integer $b$, choose $a$ to be the smallest integer such that $2^{a}>5^{b}$. By the Chinese Remainder Theorem, select $m_{0}$ to be the least positive integer satisfying the congruences $m_{0} \equiv 0\left(\bmod 2^{a}\right)$ and $6 m_{0}-1 \equiv 0\left(\bmod 5^{b}\right)$. 
TABLE 1.

\begin{tabular}{|c|c|c|c|c|c|c|c|c|c|c|c|c|c|c|}
\hline$\overline{\operatorname{gap} g}$ & $a$ & $b$ & $\begin{array}{c}\text { \# of } \\
\text { values } \\
\text { of } i\end{array}$ & $\begin{array}{c}\text { first } \\
\text { value } \\
\text { of } i\end{array}$ & gap $g$ & $a$ & $b$ & $\begin{array}{c}\text { \# of } \\
\text { values } \\
\text { of } i\end{array}$ & $\begin{array}{c}\text { first } \\
\text { value } \\
\text { of } i\end{array}$ & gap $g$ & $a$ & $b$ & $\begin{array}{c}\text { \# of } \\
\text { values } \\
\text { of } i\end{array}$ & $\begin{array}{c}\text { first } \\
\text { value } \\
\text { of } i\end{array}$ \\
\hline 1000 & 360 & 227 & 2 & 360878 & 824 & \begin{tabular}{|l|}
297 \\
\end{tabular} & 187 & 8 & 71371 & 912 & \begin{tabular}{|l|}
328 \\
\end{tabular} & 207 & 4 & 381747 \\
\hline 1002 & 360 & 7 & 3 & 548723 & 826 & 297 & 187 & 13 & 208058 & 914 & 328 & 207 & 7 & 1929 \\
\hline 1100 & 396 & 50 & 2 & 30276 & 828 & 298 & 188 & 5 & 78067 & 916 & 330 & 208 & 1 & 56173 \\
\hline 1500 & 541 & 11 & 4 & 218942 & 830 & 298 & 188 & 12 & 01315 & 918 & 330 & 208 & 4 & 45015 \\
\hline 2000 & 721 & 55 & 1 & 588652 & 832 & 300 & 189 & 8 & 53722 & 920 & 331 & 209 & 7 & 451458 \\
\hline$* 746$ & 268 & 69 & 13 & 14308 & 834 & 300 & 189 & 4 & 266194 & 922 & 331 & 209 & 8 & 31794 \\
\hline$* 748$ & 269 & 170 & 9 & 57235 & 836 & 301 & 190 & 3 & 140350 & 924 & 333 & 210 & 10 & 85698 \\
\hline$* 750$ & 269 & 70 & 6 & 364122 & 838 & 301 & 190 & 5 & 16952 & 926 & 333 & 210 & 6 & 488010 \\
\hline *752 & 271 & 71 & 14 & 44909 & 840 & 302 & 191 & 6 & 05445 & 928 & 334 & 211 & 9 & 83306 \\
\hline$* 754$ & 271 & 71 & 8 & 65890 & 842 & 302 & 191 & 9 & 74978 & 930 & 334 & 211 & 4 & 380958 \\
\hline *756 & 272 & & 7 & 41673 & 844 & 304 & 192 & 5 & 10836 & 932 & 336 & 212 & 3 & 386782 \\
\hline *758 & 272 & & 6 & 183807 & 846 & 304 & 192 & 10 & 94215 & 934 & 336 & 212 & 6 & 34178 \\
\hline *760 & 274 & 72 & 12 & 236393 & 848 & 305 & 192 & 2 & 56988 & 936 & 337 & 212 & 4 & 243622 \\
\hline *762 & 274 & 72 & 3 & 85 & 850 & 305 & 192 & 9 & 6907 & 938 & 337 & 212 & 6 & 178835 \\
\hline *764 & 275 & 73 & 3 & 253279 & 852 & 307 & 193 & 8 & 562 & 940 & 339 & 213 & 6 & 243000 \\
\hline *766 & 275 & 73 & 7 & 215388 & 854 & 307 & 193 & 9 & 38684 & 942 & 339 & 213 & 5 & 71245 \\
\hline 768 & 276 & 74 & 7 & 289399 & 856 & 308 & 194 & 7 & 119756 & 944 & 340 & 214 & 8 & 39019 \\
\hline$* 770$ & 276 & 4 & 8 & 27243 & 858 & 308 & 194 & 6 & 25518 & 946 & 340 & 214 & 5 & 8276 \\
\hline$* 772$ & 278 & & 7 & 125253 & 860 & 310 & 195 & 4 & 32742 & 948 & 341 & 215 & 3 & 507289 \\
\hline 774 & 278 & & 7 & 40978 & 862 & 310 & $1 s$ & 7 & 66748 & 950 & 341 & 215 & 5 & 59634 \\
\hline$* 776$ & 279 & 176 & 8 & 74202 & *864 & 311 & 196 & 6 & 84541 & 952 & 343 & 216 & 12 & 25613 \\
\hline *778 & 279 & 176 & 8 & 91165 & 866 & 311 & 196 & 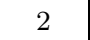 & 28141 & 954 & 343 & 216 & 5 & 19058 \\
\hline *780 & 281 & & 8 & 86527 & 868 & 313 & 197 & 13 & 7349 & 956 & 344 & 217 & 2 & 210615 \\
\hline 782 & 281 & & 9 & 21943 & 870 & 313 & 197 & 4 & 89215 & 958 & 344 & 217 & 1 & 481114 \\
\hline *784 & 282 & & 12 & 228620 & 872 & 314 & 198 & 12 & 17292 & 960 & 346 & 218 & 9 & 67752 \\
\hline 786 & 282 & & 8 & 52671 & 874 & 314 & 198 & & 9077 & 962 & 346 & 218 & 6 & 420982 \\
\hline *788 & 284 & & 5 & 225376 & 876 & 315 & 199 & 5 & 7648 & 964 & 347 & 219 & 8 & 45026 \\
\hline$* 790$ & 284 & & 13 & 53100 & 878 & 315 & 199 & 6 & 06082 & 966 & 347 & 219 & 9 & 19068 \\
\hline *792 & 285 & 180 & 7 & 147987 & *880 & 317 & 200 & 12 & 40815 & 968 & 349 & 220 & 5 & 233833 \\
\hline 794 & 285 & & 6 & 8703 & 882 & 317 & 200 & 5 & 7559 & 970 & 349 & 220 & & 75279 \\
\hline 796 & 287 & & 7 & 121321 & 884 & 318 & 201 & 6 & 214418 & 972 & 350 & 221 & 3 & 141435 \\
\hline *798 & 287 & 191 & 7 & 41947 & 886 & 318 & 201 & 5 & 49797 & 974 & 350 & 221 & 8 & 78969 \\
\hline 800 & 288 & & 12 & 18465 & 888 & 320 & 202 & 3 & 390981 & 976 & 352 & 222 & 3 & 236366 \\
\hline *802 & 288 & & 9 & 131 & 890 & 320 & 202 & 6 & 10021 & 978 & 352 & 222 & 6 & 106959 \\
\hline 04 & 289 & & 11 & 329 & 892 & 321 & 202 & 3 & & 980 & 353 & 223 & 7 & 44678 \\
\hline *806 & 289 & & 6 & & 894 & 321 & 202 & 7 & & 982 & 353 & 223 & & 17732 \\
\hline 808 & 291 & & 8 & 24033 & & 323 & 203 & 4 & & 984 & 354 & 223 & & 497244 \\
\hline 810 & 291 & & 7 & 971 & 898 & 323 & 203 & 6 & 1508 & 984 & 354 & 223 & & 497244 \\
\hline 812 & 292 & 34 & 6 & 4459 & 900 & 324 & 204 & 2 & 8389 & 986 & 354 & 223 & 6 & 86991 \\
\hline *814 & 292 & 184 & 6 & 106578 & 902 & 324 & 204 & 7 & 10429 & 988 & 356 & 224 & 9 & 36947 \\
\hline$* 816$ & 294 & 85 & 7 & 303334 & 904 & 326 & 205 & 5 & 495510 & 990 & 356 & 224 & 4 & 210425 \\
\hline 818 & 294 & $1 \zeta$ & 3 & 76118 & *906 & 326 & 205 & 3 & 3868 & 992 & 357 & 225 & 5 & 75636 \\
\hline 820 & 295 & 18 & 6 & 2834 & 908 & 327 & 206 & 2 & 1181 & 994 & 357 & 225 & 7 & 87748 \\
\hline \multirow[t]{2}{*}{822} & 295 & 186 & 5 & 324077 & 910 & 327 & 206 & 5 & 45052 & 996 & 359 & 226 & 1 & 898299 \\
\hline & & & & & & & & & & 998 & 359 & 226 & 3 & 364937 \\
\hline
\end{tabular}

Then for any $m \equiv m_{0}\left(\bmod 2^{a} 5^{b}\right)$, we have $2^{a} \mid m$ and $5^{b} \mid(6 m-1)$. Notice that $p-1=6 m, q-1=12 m-2=2(6 m-1)$, and $r-1=12 m$, so we have $2^{a}\left|(p-1), 5^{b}\right|(q-1)$, and $2^{a} \mid(r-1)$. Thus we have factorizations of part of $p-1, q-1$, and $r-1$. Now, if $m \leq 5^{3 b-1}$, then the factored parts of $p-1, q-1$, and $r-1$ are greater than $p^{1 / 3}, q^{1 / 3}$ and $r^{1 / 3}$, respectively; so we can use Theorem 1 to determine primality. 


\section{The ALGORITHM}

The following algorithm was used to find prime triples of the form $6 m+1$, $12 m-1,12 m+1$, so that the intervals $\left[96 m^{2}-2 m, 108 m^{2}+3 m-2\right]$ overlap each other and cover the entire interval $\left[10^{10}, 2^{1617}\right]$.

Step 1: Find $2^{a}, 5^{b}$ and $m_{0}$ as stated above.

Step 2: The numbers $p, q$ and $r$ that we will be testing are of the form $p=6\left(m_{0}+i 2^{a} 5^{b}\right)+1, q=12\left(m_{0}+i 2^{a} 5^{b}\right)-1, r=12\left(m_{o}+i 2^{a} 5^{b}\right)+1$, as $i$ varies. As with the prime pairs, we do not want to put all of these numbers through the primality test, since some of them may be divisible by small primes. We will sieve these numbers out as follows: For each prime $l$ up to $10^{4}$, if $i$ is in any of the residue classes

$$
\frac{-\left(6 m_{0}+1\right)}{6 \cdot 2^{a} 5^{b}}, \frac{-\left(12 m_{0}-1\right)}{12 \cdot 2^{a} 5^{b}} \text { or } \frac{-\left(12 m_{0}+1\right)}{12 \cdot 2^{a} 5^{b}} \quad(\bmod l),
$$

then either $p, q$, or $r$, respectively, will be divisible by $l$. So we will discard this value of $i$. The remaining $i$ 's we store in a table which we call $T_{1}$.

Step 3: For each value of $i$ in $T_{1}$, we use Theorem 1 to test whether $p=$ $6\left(m_{0}+i 2^{a} 5^{b}\right)+1$ is a prime. This is done in the same manner as was done for prime pairs (see Step 3 in Section 1). If we find that $p$ is prime, we perform the test with $q$. If $q$ is also prime, we then test $r$. If we have primality of $p, q$ and $r$, we proceed to Step 4 .

Step 4: Once we have a prime triple $6 m+1,12 m-1,12 m+1$, we want to find a new value, say $\tilde{m}$, so that the integers $6 \tilde{m}+1,12 \tilde{m}-1$, and $12 \tilde{m}+1$ are all prime and the intervals $\left[96 m^{2}-2 m, 108 m^{2}+3 m-2\right]$ and $\left[96 \tilde{m}^{2}-2 \tilde{m}, 108 \tilde{m}^{2}+3 \tilde{m}-2\right]$ overlap. We find $\tilde{m}$ in the following manner: We want $96 \tilde{m}^{2}<108 m^{2}$, so we need $\tilde{m} \leq \sqrt{108 / 96} m=\sqrt{9 / 8} m \approx 1.06066 \mathrm{~m}$. So we will look for $\tilde{m}$ in the range $1.03 m \leq \tilde{m} \leq 1.06 m$ by first choosing values for $a$ and $b$ such that $2^{a}, 5^{b} \approx m^{2 / 5}$. Then we let $i=\left\lfloor\frac{1.03 m}{2^{a} 5^{b}}\right\rfloor$, and select $m_{0}$ as in Step 1. Take $\tilde{m}=m_{0}+i 2^{a} 5^{b}$, as $i$ varies. Return to Step 2 and repeat.

\section{DATA}

To find prime triples of the form $6 m+1,12 m-1,12 m+1$ so that the intervals $\left[96 m^{2}-2 m, 108 m^{2}+3 m-2\right]$ overlap each other and cover the entire range $\left[10^{10}, 2^{1617}\right]$, we need $m$ to vary from about 10206 to $10^{243}$. The first prime triple found was constructed with

$$
m=8896 .
$$

To find this triple, the starting values for the exponents $a$ and $b$ of 2 and 5 were 6 and 1, respectively. This value of $m$ is less than 10206. The largest prime triple found was constructed with

$$
\begin{aligned}
m= & 8658116190552272966883810752941244739168799098740 \\
& 1254690645211635431634072427787417224738578003507 \\
& 9334280517714221366437396824089500530872622682674 \\
& 4987801486383661769150183229722484031893648332404 \\
& 29429461517315165982230003767957290013631184896 .
\end{aligned}
$$


This value of $m$ is around $10^{243}$. To find this triple, the starting values of $a$ and $b$ were 323 and 139, respectively. The algorithm described above produced over 6000 triples to cover the range $\left[10^{10}, 2^{1617}\right]$.

\section{REFERENCES}

[1] J. Brillhart, D.H. Lehmer, and J.L. Selfridge, New primality criteria and factorizations of $2^{m} \pm 1$, Math. Comp. 29:130 (1975), 620-647. MR 83j:10010

[2] A. Granville and O. Ramaré, Explicit bounds on exponential sums and the scarcity of squarefree binomial coefficients, Mathematika 43 (1996), 73-107. MR 97m:11023

[3] G.H. Hardy and J.E. Littlewood, Some problems on partitio numerorum III. On the expression of a number as a sum of primes, Acta Math. 44 (1923), 1-70.

[4] T. Nicely, New maximal prime gaps and first occurrences, Math. Comp, 68:227 (1999) 13111315. MR 99i: 11004

[5] P. Ribenboim, The new book of prime number records, Springer, New York, 1996. MR 96k:11112

[6] D. Shanks, On maximal gaps between successive primes, Math. Comp. 18 (1964), 646-651. MR 29:4745

[7] S. Weintraub, A prime gap of 864, J. Recreational Math 25:1 (1993), 42-43.

[8] J. Young and A. Potler, First occurrence prime gaps, Math. Comp. 52:185 (1989), 221-224. MR 89f:11019

Department of Mathematics, University of Georgia, Athens, Georgia 30602

Current address: Department of Mathematics, Albion College, Albion, Michigan 49224

E-mail address: PCutter@albion.edu 